\title{
A Cross-Layer Unequal Error Protection Scheme for Prioritized H.264 Video using RCPC Codes and Hierarchical QAM
}

\author{
Wei-Ho Chung*, Sunil Kumar**, Seethal Paluri**, Santosh Nagaraj**, \\ Annamalai Annamalai Jr. ${ }^{* * *}$ and John D. Matyjas ${ }^{\star * * *}$
}

\begin{abstract}
We investigate the rate-compatible punctured convolutional (RCPC) codes concatenated with hierarchical QAM for designing a cross-layer unequal error protection scheme for H.264 coded sequences. We first divide the H.264 encoded video slices into three priority classes based on their relative importance. We investigate the system constraints and propose an optimization formulation to compute the optimal parameters of the proposed system for the given source significance information. An upper bound to the significance-weighted bit error rate in the proposed system is derived as a function of system parameters, including the code rate and geometry of the constellation. An example is given with design rules for H.264 video communications and 3.5-4 dB PSNR improvement over existing RCPC based techniques for AWGN wireless channels is shown through simulations.
\end{abstract}

Keywords-Wireless Transmission, Unequal Error Protection (UEP), Rate-Compatible Punctured Convolutional (RCPC) Code, Hierarchical Modulation, H.264/AVC Video Coding

\section{INTRODUCTION}

Multimedia applications such as video streaming, which are delay sensitive and bandwidth intensive, are growing rapidly over wireless networks. However, video transmission over wireless channels requires the use of sophisticated compression techniques to reduce the data rate demands so that the available channel bandwidth can be efficiently utilized. The compressed video data is very sensitive to any bit errors or packet losses because the errors not only degrade the quality of the corrupted frame(s) but may also propagate to the subsequent frames. Wireless communication systems are prone to channel errors due to fading effects, interference, and thermal noise. These channel errors can severely degrade the quality of the received video signal.

The transmission of video over lossy wireless networks requires the use of error protection schemes against channel errors that can properly allocate system resources to minimize the dis-

\footnotetext{
※ This work was supported in part by the U.S. Department of Defense under Grant No. FA8750-08-1-0078. Approved for public release; Distribution Unlimited: 88ABW-2011-2179, 14APR11

Manuscript received September 12, 2012; accepted October 30, 2012.

Corresponding Author: Sunil Kumar

* Research Center for Information Technology Innovation, Academia Sinica, Taiwan (whc@citi.sinica.edu.tw)

** Electrical and Computer Engineering Department, San Diego State University, San Diego, CA 92182 USA

(skumar@mail.sdsu.edu, smurti1983@gmail.com, snagaraj@mail.sdsu.edu)

*** Prairie View A\&M University, TX 77446 USA (aaannamalai@pvamu.edu)

**** Air Force Research Laboratory, Rome, NY 13441 USA (john.matyjas@rl.af.mil)
} 
tortion at the receiver. In video applications (such as the H.264/AVC bit stream), some parts of the compressed video bit stream contribute more to the quality of the received video than other parts [1]. Therefore, unequal error protection (UEP) schemes are generally used to increase the robustness of multimedia transmission over wireless channels [2-5].

In this paper, we consider the state-of-the-art ISO/ITU H.264/AVC video coding standard [6, 7]. Similar to previous ITU-T and MPEG video coding standards, H.264/AVC is a block-based motion-compensated hybrid video coding scheme. H.264/AVC also has various error resiliency features for robust video transmission over lossy communication channel. Since it is difficult to recover data from the corrupted H.264/AVC video packets, and packet retransmission is usually not feasible due to real-time transmission requirements, the erroneous video packets are discarded and error concealment schemes are used [6, 8].

We use a packet prioritization scheme as described in Section II, which uses the frame type, frame location and packet data partition type information [1]. By using this prioritization scheme, the H.264 slices are distributed into three queues of different priorities based on their contribution to the received video quality. We then design a cross-layer UEP scheme for the prioritized H.264 bit stream using the joint design of RCPC codes and hierarchical QAM. The UEP scheme provides relatively higher protection, i.e., lower packet error rate (PER), to the higher priority video slices. The bit error rates (BER) of the combined RCPC code and hierarchical modulation are derived as functions of the system parameters, including the RCPC code rates and the geometry of the hierarchical QAM. As opposed to the heuristic selection of system parameters typically adopted in the literature, we propose an optimization formulation to select system parameters by pursuing the minimization of the expected loss of source significance information at the receiver. A design example using 8-QAM modulation that improves the peak signal-to-noise ratio (PSNR) of 3-priority H.264 video bit streams by nearly 3.5- $4 \mathrm{~dB}$ compared to existing RCPC based techniques over an AWGN channel is shown.

\subsection{Related Research}

Several UEP schemes have been studied in the literature. In [9-11], the BER of the hierarchical PAM and QAM are derived as functions of the geometric parameters in the constellations. In [12], a tutorial on convolutional codes is presented. In [13, 14], RCPC codes are investigated. A search for good RCPC codes was conducted, and examples of good codes were presented. Some systematic approaches of quantifying source significance information were studied in [1517]. In [18], our co-author used the asymmetric PSK modulation to minimize the distortion in pulse-coded modulation signals. In [19-20], hierarchical modulations were investigated for multimedia applications. In [2-3], joint source-channel coding and hierarchical modulation techniques were employed to optimize the perceptual video quality. In [21], the hierarchical QAM design for H.264/AVC codecs was studied. In [22], our co-author studied asymmetric 8-PSK for adaptive transmission strategies in mobile ad hoc networks. In [23], a low complexity UEP scheme using hierarchical QAM was discussed for H.264 video transmission. In [4], our coauthors demonstrated H.264 video quality improvement by using asymmetric 8-PSK modulation over Raleigh fading channels. Preliminary findings of the research discussed in this paper have been presented in [5]. 


\subsection{Novelty of the Proposed Approach}

To the best of our knowledge, the combination of forward error correction (FEC) and hierarchical modulation has not so far been investigated. When RCPC codes and hierarchical modulation are jointly employed in a system, another degree of flexibility is available. Some parts of the data can be protected only by the hierarchical modulation while others may be protected mainly by a low FEC code rate. Employing FEC codes requires constellation expansion to maintain throughput which imposes a $3 \mathrm{~dB}$ per bit penalty with QAM sets. On the other hand, hierarchical modulation improves robustness of one priority bitstream at the expense of the others, but, without any constellation expansion. So, a judicious and joint application of the two techniques has potential to outperform existing techniques.

In existing UEP schemes, the choice of system parameters (geometry of the hierarchical signal constellation) has mostly been done in a heuristic manner. For example, in [21], the modulation asymmetry parameter $\alpha$ was chosen to be either 2 or 4 heuristically. In [19], the asymmetry parameter $\theta$ was chosen adaptively based on feedback from the receiver. This requires a real-time feedback channel from the receiver which may not always exist. In [23], the asymmetry parameter $\alpha$ was chosen such that the highest priority bit error rate is fixed at $10^{-5}$, irrespective of the relative importance of the other priorities or even the channel SNR. The choice of system parameters greatly influences the UEP performance. In this paper, we systematize the choice of system parameters based on known features of the video bitstream.

Since the prioritized H.264 video bitstream is flexible as discussed in Section II, the code rate and modulation order need not be tightly constrained by the channel symbol rate. In other words, some lowest priority packets can be dropped on-the-fly, based on the available channel symbol rate under dynamic wireless channel conditions.

In Section II, we provide the background for prioritization of the video data. In Section III, we provide a brief overview of RCPC codes and hierarchical modulation. In Section IV, we describe the system model of our joint RCPC and hierarchical modulation system. In Section V, we present ways of optimizing the code and modulation parameters. We provide simulation results in Section V, and our conclusions in Section VI.

\section{Prioritization of H.264 Video Slices}

We use the H.264/AVC encoder with encoding pattern: IDR, B, P, B, P, .., I, B, P, .., B, IDR, .. The frames between two IDR (instantaneous decoding refresh) frames form one group of pictures (GOP). We have used the RTP mode of encoder where the compressed data is arranged in fixed size network abstraction layer (NAL) units which are also known as slices. Each slice can be decoded independent of other slices of the frame $[6,7]$. In this paper, we arrange each NALU or slice in a video packet and enable error resiliency features available in H.264/AVC, such as the dispersed mode of flexible macroblock ordering (FMO), constrained intra-prediction (CIP) and data partitions (DP). The data partitioning feature divides the video packets into three partitions known as DP A, DP B, and DP C [6-8].

We classify the H.264 NALUs into three priorities as shown in Table 1, which effectively utilizes the frame type and frame location within GOP, and the error resiliency features such as FMO, CIP, and data partitions. Using the dispersed mode of FMO, we divide each frame in two slice groups (SG) such that the adjacent macroblocks are assigned to slices in different SGs. 
Table 1. Slice or Packet Priority Scheme

\begin{tabular}{c|c|c|c}
\hline Priority & Frame Type & Slice Group & Data Partition \\
\hline 1 & IDR & SG1, SG2 & \\
\hline 1 & I & SG1, SG2 & DPA, DPB \\
\hline 2 & & & \\
\hline 2 & P & SG1, SG2 & DPA, DPB \\
\hline 2 & P & SG1 & DPC \\
\hline & B & SG1 & DPA \\
\hline 3 & & & DPC \\
\hline 3 & P & SG2 & DPA \\
\hline 3 & B & SG1, SG2 & DPB, DPC \\
\hline
\end{tabular}

This effectively creates a checker board-like pattern. Experiments were conducted to study the effect of complete loss of one SG data on the decoded frame quality. We observed that the error concealment scheme was able to sufficiently recover the loss of one SG when the other SG of the frame is received, due to the availability of the macroblocks surrounding the lost macroblocks. Therefore, we give higher priority to one SG over the other SG for some frame types. The CIP was enabled to decode DP B packets independent of DP C packets. Since B frames are not used as reference, and therefore do not introduce the error propagation, they are assigned a lower priority.

The IDR frames in H.264/AVC act as key frames, similar to I-frames in MPEG-4. Since the IDR frame is not partitioned by DP feature, they are assigned the highest priority ' 1 '. Since I frames (including its Data Partitions (DP) A and B) still play very important role in stopping error propagation in a GOP, they are also assigned priority ' 1 '. The DP A and DP B data of all the P frames, the DP C data of P frames in SG1, and the DP A data of B frames in SG1 is assigned priority ' 2 '. The remaining data of the GOP is assigned priority ' 3 '. We can drop the required number of the lowest priority packets if the video bit rate exceeds the channel capacity. Discussion of this packet priority scheme can be found in [1].

\section{OVERVIEW OF RCPC CODES AND HIERARCHICAL PAM/QAM}

\subsection{RCPC Codes}

In RCPC codes, a low-rate $1 / N$ convolution code is punctured with period $P$ to obtain a family of codes with rate $P /(P+l)$, where $l$ can be varied between 1 and $(N-1) P$. The puncturing of the convolutional code discards certain bits in the original low-rate mother code based on the puncturing matrix, known at both the encoder and decoder. The property of rate compatibility ensures that the bits of the high rate code are used in the low rate code. Therefore, the change of rates requires only the exchange of the puncturing matrix between the RCPC encoder and decoder $[13,14]$. By using the Viterbi decoding algorithm, the performance of RCPC code is characterized by a set of parameters, including the distance spectra $\left\{c_{k}\right\}$ and the probability of error, $P_{k}$. Here, $P_{k}$ is the probability that, in the Viterbi decoding trellis, an erroneous path deviating from the correct path by Hamming distance $k$ is selected. The BER, denoted as $P_{e, i b}$, of the information bit by using hard decision decoding is upper-bounded by 


$$
P_{e, i b}<\frac{1}{P} \sum_{k=d_{\text {free }}}^{\infty} c_{k} P_{k}
$$

\subsection{Hierarchical M-PAM}

An illustration of hierarchical $M$-PAM is shown in Fig. 1(a). The hierarchical $M$-PAM is parameterized by the set of distances which determine the geometry of the constellation points. Denoting $M=2^{m}$, the distances are represented by $\boldsymbol{d}=\left[d_{1}, d_{2}, \ldots, d_{m}\right]$. For the ordering of bits, the most significant bit (MSB) to least significant bit (LSB) is arranged from left to right. We use $b_{k}$ to denote the $k$-th bit. For example, in $b_{1} b_{2} b_{3}, b_{1}$ is the MSB and $b_{3}$ is the LSB.

We use $P_{e, P A M}\left(M, \boldsymbol{d}, b_{k}\right)$ to denote the BER of the $k$-th bit in the $M$-PAM constellation parameterized by distance vector $\boldsymbol{d}$. From [10], the BERs for $m=2$ are

$$
\begin{gathered}
P_{e, P A M}\left(4, \boldsymbol{d}, b_{1}\right)=\frac{1}{4} \operatorname{erfc}\left(\frac{d_{1}+d_{2}}{\sqrt{N_{0}}}\right)+\frac{1}{4} \operatorname{erfc}\left(\frac{d_{1}-d_{2}}{\sqrt{N_{0}}}\right), \\
P_{e, P A M}\left(4, \boldsymbol{d}, b_{2}\right)=\frac{1}{4}\left(2 \operatorname{erfc}\left(\frac{d_{2}}{\sqrt{N_{0}}}\right)-\operatorname{erfc}\left(\frac{2 d_{1}+d_{2}}{\sqrt{N_{0}}}\right)+\operatorname{erfc}\left(\frac{2 d_{1}-d_{2}}{\sqrt{N_{0}}}\right)\right),
\end{gathered}
$$

where $\operatorname{erfc}(\cdot)$ is the complementary error function. Equations (2a) and (2b) serve as roots of
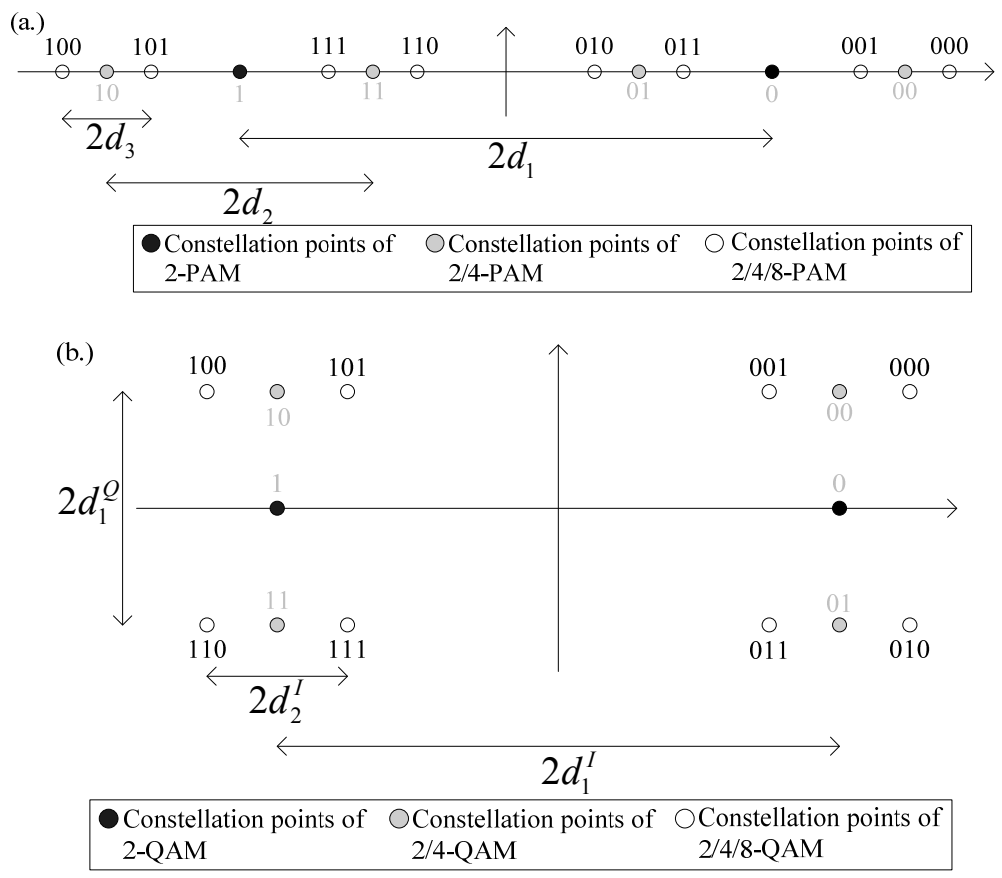

Fig. 1. Examples of constellations in hierarchical modulation, including (a) Hierarchical 2/4/8-PAM, and (b) Hierarchical 2/4/8-QAM 
the recursive algorithm to compute the BERs of higher-order hierarchical $M$-PAM. For a general higher-order $M$-PAM, i.e., $m>2$, the BER of the $k$ - $t h$ bit can be expressed by

$$
\begin{gathered}
P_{e, P A M}\left(M, \boldsymbol{d}, b_{k}\right)=\frac{1}{2} \times\left(P_{e, P A M}\left(\frac{M}{2}, \boldsymbol{d}_{+}, b_{k}\right)+P_{e, P A M}\left(\frac{M}{2}, \boldsymbol{d}_{-}, b_{k}\right)\right), \text { if } k<m, \\
P_{e, P A M}\left(M, \boldsymbol{d}, b_{k}\right)=\frac{1}{2^{m}}\left(P_{0, P A M}+P_{1, P A M}\right), \text { if } k=m,
\end{gathered}
$$

where $\boldsymbol{d}_{ \pm}=\left[d_{1}, d_{2}, \ldots, d_{m-2}, d_{m-1} \pm d_{m}\right]$, and $P_{0, P A M}$ and $P_{1, P A M}$ are described in [10].

The average energy per modulation symbol is obtained as $E_{\text {sym }}=\boldsymbol{d} \boldsymbol{d}^{T}$, where $(\cdot)^{T}$ represents the vector transposition operation.

\subsection{Hierarchical M-QAM}

We first consider hierarchical $M$-QAM with $M=2^{2 w}$ where $w$ is a positive integer. The hierarchical $M$-QAM is parameterized by a set of distances in the in-phase and quadrature components, denoted by $\boldsymbol{d}^{I}=\left[d_{1}^{I}, d_{2}^{I}, \ldots, d_{w}^{I}\right]$ and $\boldsymbol{d}^{Q}=\left[d_{1}^{Q}, d_{2}^{Q}, \ldots, d_{w}^{Q}\right]$. The example of $2 / 4 / 8-$ QAM is illustrated in Fig. 1(b). The average energy per modulation symbol can be expressed as $E_{s y m}=\boldsymbol{d}^{I}\left(\boldsymbol{d}^{I}\right)^{T}+\boldsymbol{d}^{Q}\left(\boldsymbol{d}^{Q}\right)^{T}$.

We use $P_{e, Q A M}\left(M, \boldsymbol{d}^{I}, \boldsymbol{d}^{Q}, b_{k}\right)$ to denote the BER of the $k$-th bit in $M$-QAM parameterized by distance vector $\boldsymbol{d}^{I}$ and $\boldsymbol{d}^{Q}$. The recursive approach to compute the exact BER for $M$-QAM is described in the following. First, the BER of the in-phase bits can be expressed as

$$
P_{e, Q A M}\left(M, \boldsymbol{d}^{I}, \boldsymbol{d}^{Q}, b_{k}\right)=P_{e, P A M}\left(\sqrt{M}, \boldsymbol{d}^{I}, b_{k}\right),
$$

for $k=1,2, \ldots, \frac{1}{2} \log _{2} M$.

Then, BER of the quadrature bits can be expressed as

$$
P_{e, Q A M}\left(M, \boldsymbol{d}^{I}, \boldsymbol{d}^{Q}, b_{k}\right)=P_{e, P A M}\left(\sqrt{M}, \boldsymbol{d}^{Q}, b_{k}\right)
$$

For $M=2^{2 w+1}$, the distance vectors are $\boldsymbol{d}^{I}=\left[d_{1}^{I}, d_{2}^{I}, \ldots, d_{w+1}^{I}\right]$ and $\boldsymbol{d}^{Q}=\left[d_{1}^{Q}, d_{2}^{Q}, \ldots, d_{w}^{Q}\right]$. The BER of the in-phase bits can be expressed as

$$
P_{e, Q A M}\left(M, \boldsymbol{d}^{I}, \boldsymbol{d}^{Q}, b_{k}\right)=P_{e, P A M}\left(\sqrt{2 M}, \boldsymbol{d}^{I}, b_{k}\right)
$$

for $k=1,2, \ldots, \frac{1}{2} \log _{2} 2 M$.

The BER of the quadrature bits can be expressed as

$$
P_{e, Q A M}\left(M, \boldsymbol{d}^{I}, \boldsymbol{d}^{Q}, b_{k}\right)=P_{e, P A M}\left(\sqrt{M / 2}, \boldsymbol{d}^{Q}, b_{k}\right),
$$


for $k=1,2, \ldots, \frac{1}{2} \log _{2} \frac{M}{2}$

By using (4) and (5), the BERs of the $M$-QAM can be computed recursively for each bit position.

\section{Proposed Cross-Layer System Model}

The proposed framework is composed of RCPC codes followed by the hierarchical PAM/QAM, as illustrated in Fig. 2. The source generates $N$ prioritized bit streams. The significance of the $k$-th stream is $f_{S S I}\left(b_{k}\right)$ and its data rate is $r_{k}$. The code rates of the RCPC codes are denoted by $\left\{R_{1}, R_{2}, \ldots, R_{N}\right\}$, where, $R_{k}$ represents the RCPC code rate for the $k$-th priority bit stream. The RCPC code rate is expressed as $R_{k}=P /\left(P+l_{k}\right)$ where $\mathrm{P}$ is the period of the mother convolutional code and $l_{k}$ is the length of the redundancy bits. The channel symbol rate is $C$ symbols/sec. The net data transmission rate of the $k$-th priority data stream is $C R_{k}$.

Some low priority packets might have to be dropped at the source itself if the total symbol rate of the bitstream is higher than C. Since the prioritized H.264 bit stream can tolerate loss of some lowest priority packet as discussed in Section II, the code rate and modulation order need not be tightly constrained by the channel symbol rate.

We denote $E_{b}$ as the average energy per bit and $E_{\text {sym }}$ as the average energy per modulation symbol. The average energy per information bit can be expressed as

$$
E_{b}=\frac{E_{s y m} C}{r_{1}+r_{2}+\ldots+r_{m}} .
$$
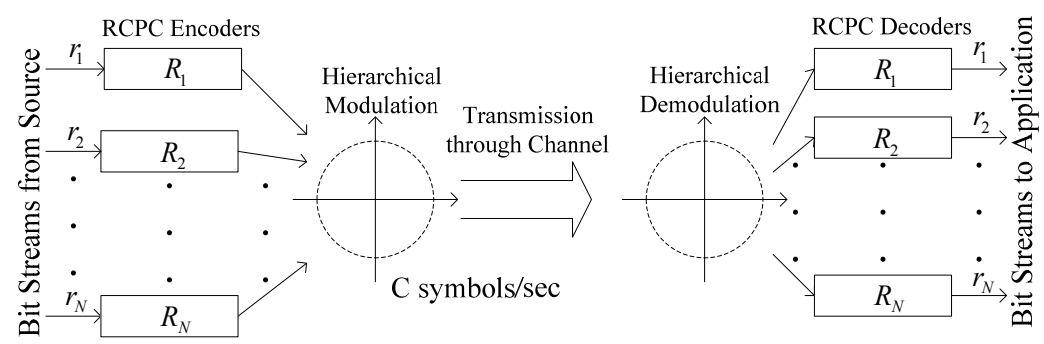

Fig. 2. System model of the proposed joint RCPC and hierarchical modulation system

\subsection{Bit Error Rate of RCPC Codes in Binary Symmetric Channel}

In RCPC codes, the BER of the information bit, denoted by $P_{e, i b}$, by using hard decision decoding was given in (1). The union-bound in (1) can be truncated after only a few terms at moderate-to-high signal to noise ratio (SNR). The distance spectrum can be obtained from the transfer function of the mother convolutional code and the puncturing matrix. The pairwise error probability terms $P_{k}$ can be expressed as [12]: 


$$
\begin{aligned}
& P_{k} \\
& =\sum_{h=(k+1) / 2}^{k}\left(\begin{array}{l}
k \\
h
\end{array}\right) p^{h}(1-p)^{k-h}, \text { if } k \text { odd } \\
& =\frac{1}{2}\left(\begin{array}{c}
k \\
k / 2
\end{array}\right) p^{k / 2}(1-p)^{k / 2}+ \\
& \sum_{h=k / 2+1}^{k}\left(\begin{array}{l}
k \\
h
\end{array}\right) p^{h}(1-p)^{k-h}, \text { if } k \text { even }
\end{aligned}
$$

where, $p$ is the BER for the coded bit of the RCPC. Since the RCPC is concatenated with the hierarchical PAM/QAM, $p$ is now the BER of the specific hierarchical PAM/QAM bit. For example, the $p$ for RCPC with $M$-PAM can be computed by (2) and (3), and the $p$ for RCPC with $M$-QAM can be computed by (4) and (5).

\subsection{Aggregation of Modulation Bits}

We select the order of the modulation to be equal or larger than the number of priorities. In other words, with a modulation of $M=2^{m}$ points and $N$ priorities, we need $m \geq N$. Since $m$ $<N$ necessitates complex mapping from the prioritized bitstreams to modulation labels rendering real-time adaptation impractical, we do not use it in this paper. We can consider the bank of $N$ RCPC codes as $N$ channels to transport the $N$ prioritized bit streams. Each channel transports the bit stream of a single priority. With the modulation order $M=2^{m}$, the modulation scheme is capable of transporting $m$ bit streams. Using the RCPC codes concatenated to the modulation scheme, the output bit streams enter as the input bit streams to the hierarchical PAM/QAM. Therefore, in the case of $m=N$, i.e., when the number of source bit streams is equal to the number of bit streams in the modulation, each bit stream is transported by a single channel in the modulation scheme. In the case of $m>N$, where the number of source bit streams is smaller than the number of bit streams in the modulation, an aggregation of modulation bit streams is performed to transport the $N$ source bit streams as

$$
\left\{\gamma_{k}, k=1 \sim N, \sum_{k=1}^{N} \gamma_{k}=m\right\} .
$$

Here $\gamma_{k}$ represents the number of modulation bit streams aggregated to collectively transport the $k^{\text {th }}$ source bit stream. The aggregation is conducted by matching the order of importance in the source bit streams to the order of significance in the modulation bit streams.

\subsection{Bit Error Rate for RCPC Code with Hierarchical Modulation}

We denote the modulation scheme as $\Omega \in\{P A M, Q A M\}$, distance vector as $D$, and the BER of the RCPC coded bit of the $k$-th bit stream as $p_{e, c b, k}$. With the aggregation of modulation bit streams by $\left\{\gamma_{k}\right\}$, the BER of the code bit at the $k$-th bit stream can be expressed as 


$$
p_{e, c b, k}=\frac{1}{\gamma_{k}} \sum_{\forall b_{h} \in \gamma_{k}} P_{e, \Omega}\left(M, D, b_{h}\right) .
$$

Therefore, an upper bound to the BER of information bits at the $k$-th source bit stream, denoted as $P_{e, i b, k}$, can be derived by using (1), (6) and (7) and replacing the $p$ in (6) by the $p_{e, c b, k}$ in (7). Thus, $P_{e, i b, k}$ is a function of the modulation type $\Omega$, modulation order $M$, signal constellation distances $D$, and the aggregation $\left\{\gamma_{k}\right\}$, and can be expressed as $p_{e, i b, k}\left(\Omega, M, D,\left\{\gamma_{h}\right\}\right)$.

\section{Optimization of Parameters}

In this section, we derive a mathematical formulation to compute the optimum parameters of the proposed combined RCPC codes with hierarchical QAM. Constraints of the system are:

1.The transmission rate must be smaller or equal to the source information bit rate for each priority, i.e., $r_{k} \geq R_{k} \gamma_{k} C, k=1 \sim N$; the inequality allows some packets to be dropped at the source itself.

2.The code rates $\left\{R_{k}\right\}$ must be feasible;

3.The transmitted bit energy $E_{b}$ must be less than $E_{b, \max }$, i.e., $\frac{E_{s y m} C}{r_{1}+r_{2}+\ldots+r_{m}}<E_{b, \max }$.

For a set of source significance information $f_{S S I}\left(b_{k}\right)$, the objective is to minimize the expected loss of data significance information at the receiver, written as

$$
\min _{\Omega, M, D, A, \Re} \sum_{k=1}^{N} f_{S S I}\left(b_{k}\right) P_{e, i b, k}(\Omega, M, D, A, \Re) .
$$

This formulation represents a generic expression to select the system parameters. In the scenarios where some of the system parameters are fixed, the parameter set of the objective function in (8) may be a subset of $\left\{\Omega, M, D,\left\{\gamma_{k}\right\}\right\}$. This problem can be solved by using the genetic algorithms which are computationally complex. We propose below a suboptimal solution using some approximations, in order to reduce the computational complexity of our optimization formulation.

The information bit error probability expressions $P_{e, i b, k}(M, \boldsymbol{d})$ can be simplified in some cases to allow for a simpler computation of the optimum system parameters. For example, by ignoring non-nearest neighbors in the 4PAM signal constellation, equations (2a) and (2b) can be simplified as

$$
\begin{aligned}
& P_{e, P A M}\left(4, \boldsymbol{d}, b_{1}\right)=\frac{1}{4} \operatorname{erfc}\left(\frac{d_{1}-d_{2}}{\sqrt{N_{0}}}\right), \\
& P_{e, P A M}\left(4, \boldsymbol{d}, b_{2}\right)=\frac{1}{4} \operatorname{erfc}\left(\frac{2 d_{1}-d_{2}}{\sqrt{N_{0}}}\right)+\frac{1}{2} \operatorname{erfc}\left(\frac{d_{2}}{\sqrt{N_{0}}}\right) .
\end{aligned}
$$


The second derivative matrices (the Hessians) of the above BER expressions can be shown to be

$$
\begin{aligned}
& H\left(b_{1}\right)=\frac{\left(d_{1}-d_{2}\right)}{N_{0}} \exp \left(\frac{\left(d_{1}-d_{2}\right)^{2}}{2 N_{0}}\right)\left[\begin{array}{cc}
1 & -1 \\
-1 & 1
\end{array}\right], \\
& H\left(b_{2}\right)=\frac{\left(2 d_{1}-d_{2}\right)}{N_{0}} \exp \left(\frac{\left(2 d_{1}-d_{2}\right)^{2}}{2 N_{0}}\right)\left[\begin{array}{cc}
4 & -2 \\
-2 & 1
\end{array}\right],
\end{aligned}
$$

which are both positive semi-definite if $d_{1}>d_{2}$ (which is always satisfied for hierarchical 4PAM), and have different eigen-vectors corresponding to their zero eigen-values. The objective function in (8) has a Hessian that is positive definite since it is a linear combination of the two Hessians above. This objective function is therefore convex. The resulting formulation can be solved using standard convex optimization techniques such as the interior-point method. This simplified convex formulation facilitates faster system design and therefore real time implementation. However, it may not be possible to obtain generic convex approximations that are valid for all M-QAM sets due to the recursive nature of equations (3a) and ( $3 b$ ).

\section{Simulation RESULtS}

Our simulations demonstrate the improvement in PSNR when transmitting H.264 video data over noisy channels using the proposed scheme. We have used the CIF Foreman and Silent video test sequences (352x288 pixels, RGB color components with 24 bits per pixel, 300 frames) encoded by H.264/AVC encoder using the JM14.2 Extended Profile at 512kbps and 30 frames/second with 150 Byte NALU size packets. The H.264 NALUs or packets are divided into three priorities based on their frame and data partition type as shown in Table 1. Packets of each priority are arranged in a separate queue - thus resulting in three queues - and protected using the prioritized RCPC code with hierarchical 8-QAM.

With slight traffic shaping, the traffic ratio of the three priorities is 15:20:24. The source significance information is $f_{S S I}\left(b_{k}\right)=12,4$, and 2 for $k=1,2$, and 3 , respectively. Here $k$ represents the priority class with ' 1 ' being the highest priority and ' 3 ' being the lowest. This source significance information was computed based on the distortion in the received video quality (i.e., mean squared error (MSE) values) caused by the loss of packets in each priority. We use the RCPC code given in Table I of [14] with $P=8$. The resulting RCPC code rates for the three priorities after optimization are $R_{1}=8 / 16, R_{2}=8 / 12, R_{3}=8 / 10$. We used an additive white Gaussian noise (AWGN) channel with the received SNR set to $10 \mathrm{~dB}$. The system parameters were obtained by minimizing the expected loss of utility, formulated as,

$$
\min _{D} \sum_{k=1}^{3} f_{S S I}\left(b_{k}\right) P_{e, i b, k}\left(Q A M, 8, D,\left\{\gamma_{h}=1\right\}\right)
$$

This optimization was performed using a genetic algorithm, which generates the optimal parameter set as $\boldsymbol{d}^{I}=[7.11,4.31]$ and $\boldsymbol{d}^{Q}=[3.85]$, which are denoted as Optimized Parameters in Fig. 3 and 4. For comparison, $\boldsymbol{d}^{I}=[7.2,4.2]$ and $\boldsymbol{d}^{Q}=[3.8]$ are selected as Parameter 


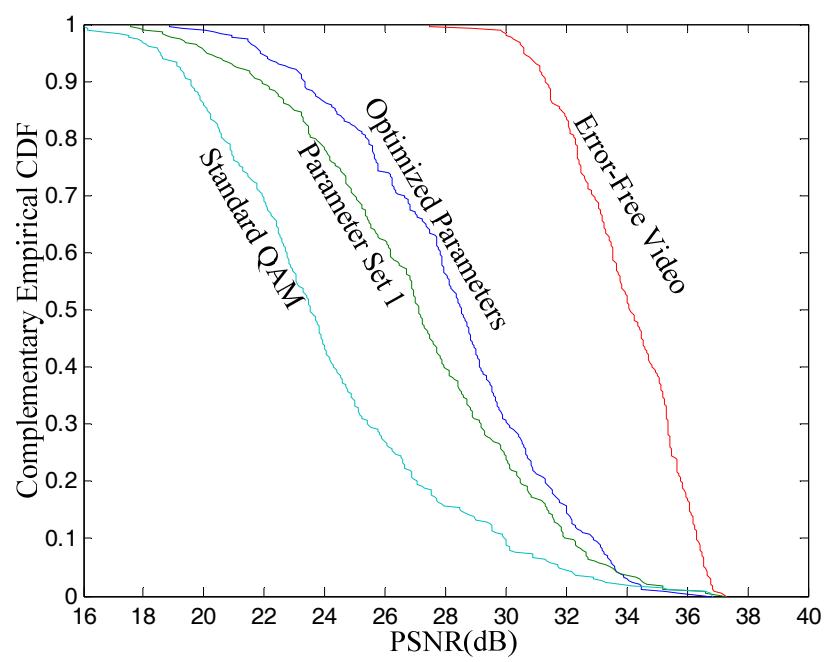

Fig. 3. Complementary empirical CDFs of the per-frame PSNRs in Foreman video sequence. The four curves are complementary CDFs of PSNRs in reconstructed Foreman videos transmitted by the error-free transmission, optimized parameter, parameter set 1, and standard QAM

Set 1 whose values are slightly different from the optimal. The PSNRs of the transmitted and reconstructed videos are computed on a per-frame basis and the empirical cumulative distribution functions (CDF) are shown in Fig. 3 (for Foreman video) and Fig. 4 (for Silent video). From Figs. 3 and 4, we can see that the PSNR of the received video is highest with the optimal param-

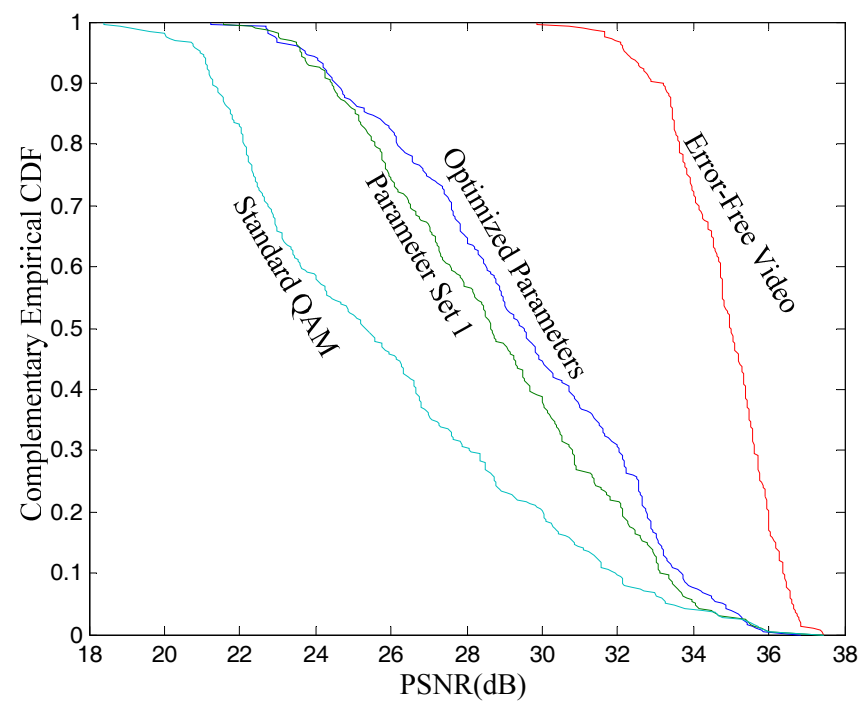

Fig. 4. Complementary empirical CDFs of the per-frame PSNRs in Silent video sequence. The four curves are complementary CDFs of PSNRs in reconstructed Silent videos transmitted by the error-free transmission, optimized parameter, parameter set 1, and standard QAM 
eter set. Specifically, the average PSNR in Fig. 3 for the error-free case, optimal set, Parameters Set 1 and the standard (symmetric) QAM are $33.5 \mathrm{~dB}, 26.8 \mathrm{~dB}, 25.3 \mathrm{~dB}$, and $22.6 \mathrm{~dB}$, respectively. In Fig. 4, the average PSNRs for the error-free cases, optimal set, parameters set 1 and the standard QAM are $34.6 \mathrm{~dB}, 28.1 \mathrm{~dB}, 27.6 \mathrm{~dB}$, and $24.6 \mathrm{~dB}$ respectively. Therefore, the use of asymmetric 8-QAM achieves up to 3.5 - $4 \mathrm{~dB}$ PSNR improvement as compared to the standard 8-QAM with symmetric constellation. We have used the same set of optimum RCPC code rates, i.e., $\{8 / 16,8 / 12,8 / 10\}$, for the optimized parameters, parameter set 1 , and standard 8 QAM in transmitting the video data.

The reconstructed frames with each technique for Foreman and Silent video sequences are compared in Figs. 5 and 6, respectively. Comparing the reconstructed frames from the Optimal

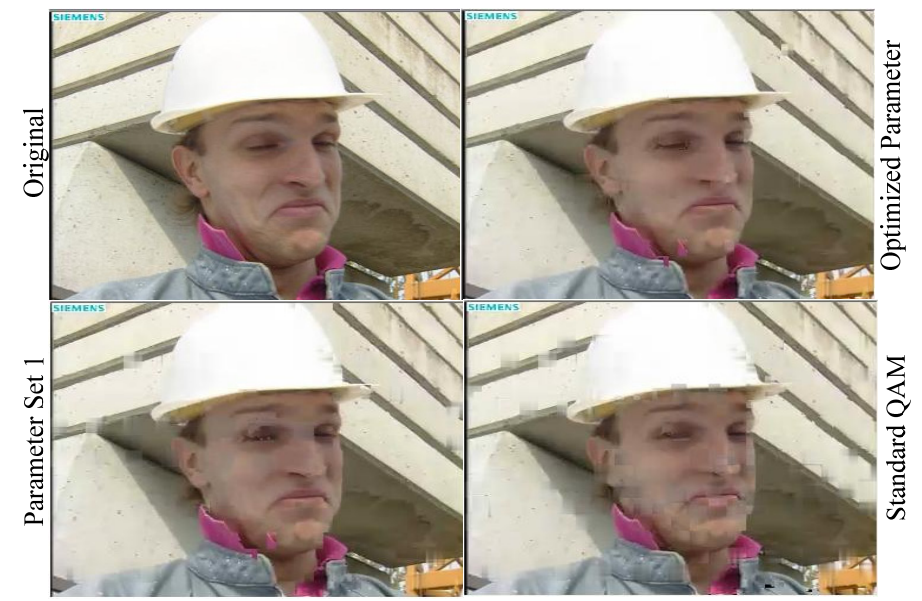

Fig. 5. The original (top left) and reconstructed frames of the Foreman video, using optimized parameters (top right), parameter set 1 (bottom left), and standard QAM (bottom right)

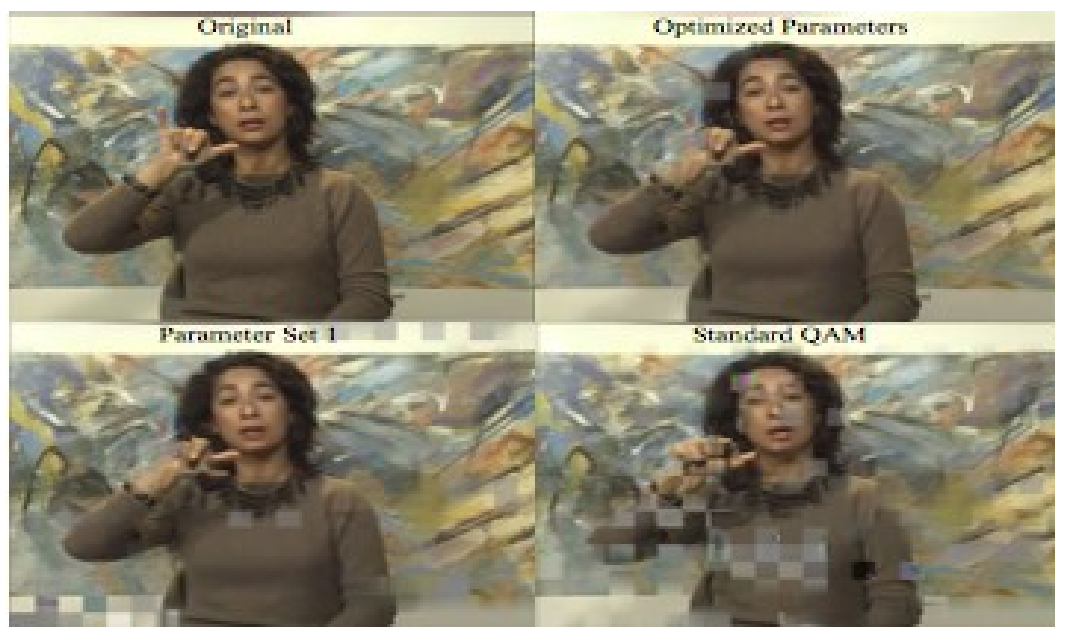

Fig. 6. The original and reconstructed frames of the Silent video 
Parameter set and Parameter Set 1 in Figs. 5 and 6, we observe that slight deviations in the parameters set cause visible differences, while the reconstructed frame from standard QAM shows the worst distortion. Therefore, the effectiveness of the proposed scheme and optimization formulation are verified. Our simulations at other channel SNRs showed similar benefits with the proposed joint hierarchical modulation and RCPC encoding system.

\section{ConClusions}

In this paper, we proposed a novel framework combining RCPC codes and hierarchical PAM/QAM to achieve unequal error protection for H.264 video. The H.264 data was divided into three priority queues by utilizing the relative importance of the data packets in the GOP. While heuristic choices of systems parameters are often employed in conventional unequal error protection schemes, we investigated optimizing the selection of parameters in the proposed system. By using the upper bound on the BERs and the available source significance information, we obtained the optimum system parameters by minimizing the expected loss of data significance information at the receiver. The proposed joint design improved the quality of video communications significantly by utilizing useful aspects of both asymmetric modulation and code rate adaptation.

\section{REFERENCES}

[1] S. Kumar, A. Janarthanan, M. M. Shakeel, S. Maroo, John D. Matyjas and M. Medley, "Robust H.264/AVC Video Coding with Priority Classification, Adaptive NALU Size and Fragmentation," IEEE MILCOM, 2009, Boston, USA.

[2] S. Arslan, P. Cosman and L. B. Milstein, "Progressive source transmissions using joint source-channel coding and hierarchical modulation in packetized networks," IEEE Globecom, 2009.

[3] T.-L. Lin and P. Cosman, "Perceptual video quality optimization in AWGN channel using low complexity channel code rate allocation," Asilomar Conf. on Signals, Systems and Computers, 2009.

[4] A. Bhatnagar, S. Kumar, A. Janarthanan, A. Annamalai Jr, M. Medley and J. Matyjas, "A Multilevel Unequal Protection Scheme for Robust H.264/AVC Video Transmission over Wireless Channels," IEEE MILOCM, Boston, USA, October, 2009.

[5] W.-H. Chung, S. Paluri, S. Kumar, S. Nagaraj and John D. Matyjas, "Unequal Error Protection for H.264 Video using RCPC Codes and Hierarchical QAM," IEEE ICC, May 23 - 27, 2010, Cape Town, S. Africa.

[6] T. Wiegand, G.J. Sullivan, G. Bjontegaard, A. Luthra, "Overview of the H.264/AVC video coding standard," IEEE Trans. CSVT, Vol.13(7), July, 2002, pp.560-576.

[7] "Draft ITU-T Recommendation and Final Draft International Standard of Joint Video Specification (ITU-T Rec. H.264/ISO/IEC 14 496-10 AVC," In Joint Video Team (JVT) Of ISO/IEC MPEG and ITU-T VCEG, JVTG050, 2003.

[8] S. Kumar, L. Xu, M. K. Mandal and S. Panchanathan, "Error Resiliency Schemes in H.264/AVC Standard," Elsevier J. Visual Communication \& Image Representation (Special issue on Emerging H.264/AVC Video Coding Standard), Vol.17(2), 2006, pp.425-450.

[9] P. K. Vitthaladevuni and M.-S. Alouini, "A closed-form expression for the exact BER of generalized PAM and QAM constellations," IEEE Trans. Commun., Vol.52 (5), May, 2004, pp.698-700. 
[10] P. K. Vitthaladevuni and M.-S. Alouini, "A recursive algorithm for the exact BER computation of generalized hierarchical QAM constellations," IEEE Trans. Inf. Th., Vol.49(1), January. 2003, pp.297307.

[11] P. K. Vitthaladevuni and M.-S. Alouini, "BER computation of 4/M-QAM hierarchical constellations," IEEE Trans. Broadcast., Vol.47(3), 2001, pp.228-239.

[12] A. Viterbi, "Convolutional Codes and Their Performance in Communication Systems," IEEE Trans. Commun., Vol.19(5), Part 1, October. 1971, pp.751-772.

[13] L. H. C. Lee, "New rate-compatible punctured convolutional codes for Viterbi decoding," IEEE Trans. Commun., Vol.42(12), December. 1994, pp.3073-3079.

[14] J. Hagenauer, "Rate-compatible punctured convolutional codes (RCPC codes) and their applications," IEEE Trans. Commun., Vol.36(4), April, 1988, pp.389-400.

[15] N. Goertz, T. Eriksson, J. B. Anderson, "Logarithmic Bit-Significance Ratio: Definition, Calculation Rules and Examples,” IEEE Symp. Inf. Th., 2006, pp.2869-2873.

[16] P. Poda, A. Tamtaoui and E. H. Bouyakhf, "Designing DCT Source Significance Information for Efficient and Robust Transmission," Contemporary Engineering Sciences, Vol.2(2), 2009, pp.67-84.

[17] M.G. Martini and M. Chiani, "Rate-distortion models for unequal error protection for wireless video transmission," 59th IEEE VTC, Vol.2, 2004, pp.1049-1053.

[18] J. Lu, A. Annamalai, D. R. Vaman, "Asymmetric PSK Constellation Design to Minimize Distortion in PCM Data Transmission," IEEE Sarnoff Symp., April, 2008, pp.1-5.

[19] M. B. Pursley and J. M. Shea, "Adaptive Non-uniform Phase-Shift-Key Modulation for Multimedia Traffic in Wireless Networks," IEEE J. Sel. Areas Commun., Vol.18(8), August, 2000, pp.1394-1407.

[20] M. Morimoto, M. Okada, and S. Komaki, "A hierarchical image transmission system in a fading channel," IEEE Int. Conf. Universal Personal Commun., 1995, pp.769-772.

[21] M. M. Ghandi, M. Ghanbari, "Layered H.264 video transmission with hierarchical QAM," Elsevier J. Visual Commun. and Image Representation, Vol.17(2), 2006.

[22] A. Annamalai, Jing Lu, D. R. Vaman, "Improving the Efficiency of Wireless Networks via a Passive Rate-Adaptation Strategy," Wireless Telecom Symp.,CA, 2008.

[23] Y. Chang, S. Lee, and R. Komiya, "A low-complexity unequal error protection of H.264/AVC video using adaptive hierarchical QAM," IEEE Trans. Consumer Electronics, Vol.52(4), November, 2006, pp.1153-1158.

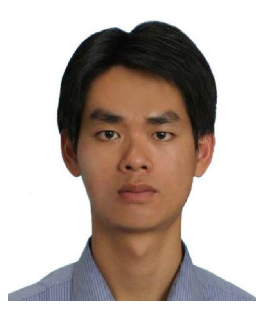

\section{Wei-Ho Chung}

He received a Ph.D. degree in the Electrical Engineering Department at University of California, Los Angeles, USA, in 2009. In 2009, he was a research associate at San Diego State University. Since January 2010, Dr. Chung has been a faculty member in the Research Center for Information Technology Innovation, Academia Sinica, Taiwan. His research interests include wireless communications, signal processing, statistical detection and estimation theory, and networks. 


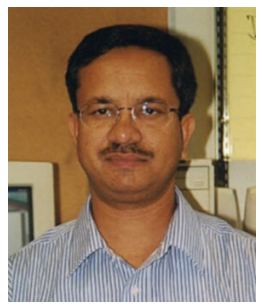

\section{Sunil Kumar}

He received a Ph.D. (Electrical and Electronics Engineering) from the Birla Institute of Technology and Science, Pilani (India) in 1997. From 1997 to 2002, he was a Postdoctoral Researcher in the Integrated Media Systems Center at University of Southern California, Los Angeles. From 2002 to 2006, he was an Assistant Professor in the Electrical and Computer Engineering department at Clarkson University, NY. Since August 2006, he has been an Associate Professor and Thomas G. Pine Faculty Fellow in the Electrical and Computer Engineering department at San Diego State University, CA. His research interests include robust video over wireless and QoS-aware protocols for multimedia traffic in wireless networks. He has published 120 research articles in journals and conferences, and one book.

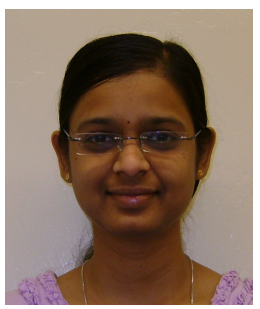

\section{Seethal Paluri}

She received her B.S. in Electrical and Computer Engineering from University of the West Indies, Trinidad and Tobago in 2005 and her M.S. degree in Electrical Engineering from University of Southern California, Los Angeles in 2008. She is currently pursuing her doctoral degree in Computational Sciences at San Diego State University. Her research interests are in the areas of error resilient image and video compression. She also works on developing statistical modeling techniques for video quality prediction and prioritization for reliable real-time QoS aware streaming over wireless networks.

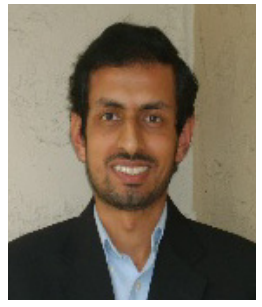

\section{Santosh Nagaraj}

He received his PhD from Purdue University, West Lafayette, IN in 2005. Currently, he is an Associate Professor of Electrical and Computer Engineering at the San Diego State University, San Diego, CA. His research interests are primarily in the areas of communication system design, broadband modulation and demodulation techniques, and signal processing.

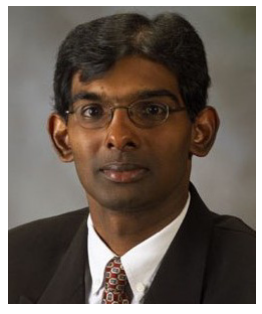

\section{Annamalai Annamalai Jr.}

$\mathrm{He}$ is a Professor of Electrical Engineering at the Prairie View A\&M University. He has held faculty appointment at Virginia Tech (1999-2006). Dr. Annamalai has published over 150 peer-reviewed articles and five book chapters in the field of wireless communications. He won the 2001 IEEE Leon Kirchmayer Prize Paper award, and the IEEE VTS/Motorola Daniel E. Noble Fellowship. He has served on the Editorial Board of four IEEE journals/transactions and the Technical Program Chair of the 2002 IEEE VTC. His research interests include crosslayer design and optimization of cooperative wireless communications and cognitive radio networks. 


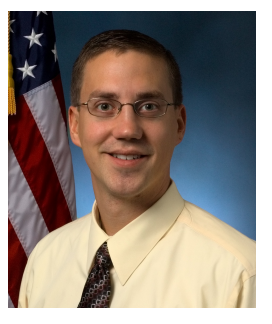

John Matyjas

He received his Ph.D. in Electrical Engineering from the State University of New York (SUNY) at Buffalo in 2004. Currently, he is a senior scientist with the Air Force Research Laboratory in Rome, NY. His research interests are in the areas of cognitive wireless multiple-access communications and networking, statistical signal processing and optimization, and neural networks. Dr. Matyjas is recipient of the 2010 IEEE ICC Best Paper Award, 2009 Mohawk Valley Engineering Executive Council "Engineer of the Year" Award and the 2009 Fred I. Diamond Basic Research Award for "best technical paper." 